\title{
Ultrafast dynamics of an unoccupied surface resonance state in $\mathrm{Bi}_{2} \mathrm{Te}_{2} \mathrm{Se}$
}

\author{
Munisa Nurmamat, ${ }^{1}$ E. E. Krasovskii, ${ }^{2,3,4}$ Y. Ishida, ${ }^{5}$ K. Sumida, ${ }^{1}$ Jiahua Chen, ${ }^{1}$ T. Yoshikawa, ${ }^{1}$ E. V. Chulkov, ${ }^{2,3}$ \\ K. A. Kokh, ${ }^{6,8}$ O. E. Tereshchenko, ${ }^{7,8}$ S. Shin, ${ }^{5}$ and Akio Kimura ${ }^{1}$ \\ ${ }^{1}$ Department of Physical Sciences, Graduate School of Science, Hiroshima University, 1-3-1 Kagamiyama, \\ Higashi-Hiroshima 739-8526, Japan \\ ${ }^{2}$ Departamento de Física de Materiales UPV/EHU, CFM-MPC UPV/EHU, 20080 San Sebastían/Donostia, Basque Country, Spain \\ ${ }^{3}$ Donostia International Physics Center (DIPC), 20018 San Sebastían/Donostia, Basque Country, Spain \\ ${ }^{4}$ IKERBASQUE, Basque Foundation for Science, 48013 Bilbao, Spain \\ ${ }^{5}$ Institute for Solid State Physics, the University of Tokyo, 5-1-5 Kashiwa-no-ha, Kashiwa, Chiba 277-8581, Japan \\ ${ }^{6}$ V. S. Sobolev Institute of Geology and Mineralogy, Siberian Branch, Russian Academy of Sciences, Koptyuga Prospekt 3, \\ Novosibirsk 630090, Russia \\ ${ }^{7}$ A. V. Rzhanov Institute of Semiconductor Physics, Siberian Branch, Russian Academy of Sciences, Prospekt Akademika Lavrentieva 13, \\ Novosibirsk 630090, Russia \\ ${ }^{8}$ Novosibirsk State University, Ulica Pirogova 2, Novosibirsk 630090, Russia
}

(Received 5 February 2018; published 8 March 2018)

\begin{abstract}
Electronic structure and electron dynamics in the ternary topological insulator $\mathrm{Bi}_{2} \mathrm{Te}_{2} \mathrm{Se}$ are studied with time- and angle-resolved photoemission spectroscopy using optical pumping. An unoccupied surface resonance split off from the bulk conduction band previously indirectly observed in scanning tunneling measurements is spectroscopically identified. Furthermore, an unoccupied topological surface state (TSS) is found, which is serendipitously located at about $1.5 \mathrm{eV}$ above the occupied TSS, thereby facilitating direct optical transitions between the two surface states at $\hbar \omega=1.5 \mathrm{eV}$ in an $n$-type topological insulator. An appreciable nonequilibrium population of the bottom of the bulk conduction band is observed for longer than $15 \mathrm{ps}$ after the pump pulse. This leads to a long recovery time of the lower TSS, which is constantly populated by the electrons coming from the bulk conduction band. Our results demonstrate $\mathrm{Bi}_{2} \mathrm{Te}_{2} \mathrm{Se}$ to be an ideal platform for designing future optoelectronic devices based on topological insulators.
\end{abstract}

DOI: 10.1103/PhysRevB.97.115303

\section{INTRODUCTION}

Three-dimensional topological insulators (TIs) are an exotic quantum phase of matter with an insulating bulk and a conducting surface. The robust topologically protected Dirac fermions with helical spin texture give rise to unique transport properties [1-6]. Direct observation of the electronic structure and ultrafast dynamics of the nonequilibrium carriers well above the Fermi level is important for spintronic applications, which has brought about many pump-probe studies of the related materials [7-16].

It is essential to understand the interplay between the bulk and the surface states because the recovery of the equilibrium population depends on how strongly they couple to each other $[11,12]$. The unoccupied surface resonance state (SRS) split off from the bulk conduction band was identified in a first-principles calculation in our previous work on $\mathrm{Bi}_{2} \mathrm{Te}_{2} \mathrm{Se}$ (BTS), where it was drawn on to explain the spin-selective scattering of the topological surface state (TSS) [17]. Recently, a surface resonance was directly observed in $\mathrm{Bi}_{2} \mathrm{Se}_{3}$ by timeand angle-resolved photoemission spectroscopy (TARPES) also with spin resolution (STARPES), and its spin texture was studied both experimentally and theoretically $[18,19]$. These findings are important to reveal the relationship between TSS and SRS. However, the carrier dynamics of photoexcited electrons in SRS and its effect on the ultrafast relaxation processes have not yet been clarified.
The newly discovered unoccupied TSS in $\mathrm{Bi}_{2} \mathrm{Se}_{3}$ located $1.5 \mathrm{eV}$ above the conduction band minimum allows a direct photoexcitation of the electrons in the $n$-type semiconductor by the radiation of the Ti:sapphire femtosecond laser [11]. The population of the unoccupied TSS by the excitation of the occupied TSS with the 1.5-eV photons is expected to bring unique information about the nonequilibrium electron dynamics at the surface, which cannot be inferred from studying the spin-degenerate conduction bands. Here, the advantage stems from the topological character and the similar helical spin texture of the TSS, which is not the case in the photogalvanic effect in the spin-degenerate bulk conduction band, where the spin polarization of the electrons optically excited from the TSS is significantly reduced [20]. This makes the studies of the nonequilibrium surface electrons indispensable for the development of TI-based optical devices.

In this work, we focus on a particularly promising ternary compound, BTS, in which the bulk conductivity is strongly suppressed because of its unique atomic arrangement [21], which is in contrast to the more popular binary TI systems, such as $\mathrm{Bi}_{2} \mathrm{Se}_{3}$ and $\mathrm{Bi}_{2} \mathrm{Te}_{3}$, where the contribution from the surface states is blurred by the dominant bulk carriers [22-24]. In our previous scanning tunneling microscopy/spectroscopy study on BTS [17] a strong warping of the constant energy contours (CECs) of the lower TSS was shown to lead to an anisotropic spin-selective scattering in the unoccupied part. 

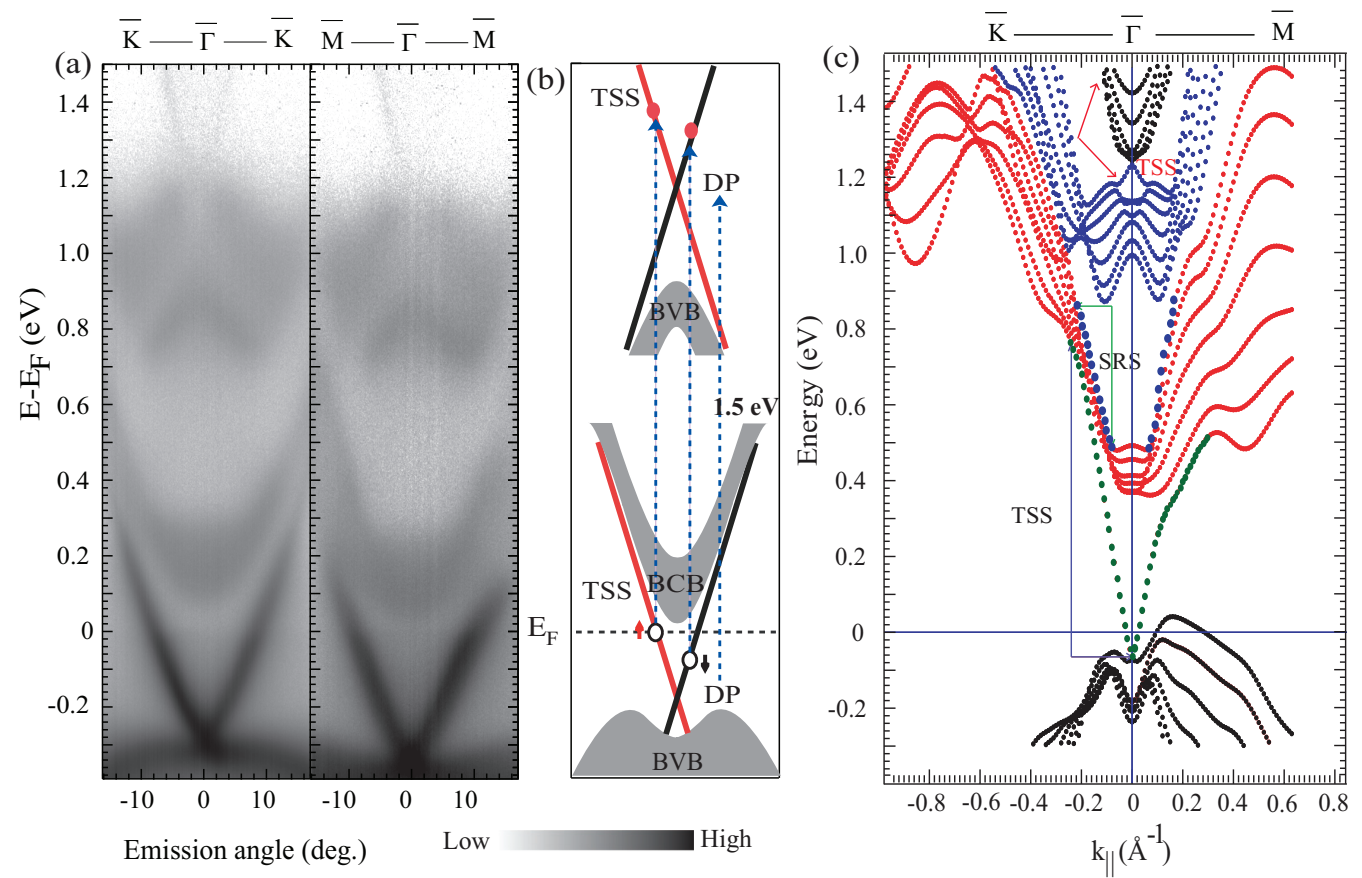

FIG. 1. (a) TARPES spectrum of $\mathrm{Bi}_{2} \mathrm{Te}_{2}$ Se taken after pumping along the $\bar{\Gamma} \bar{K}$ and $\bar{\Gamma} \bar{M}$ directions. (b) Schematic illustration of the direct optical transition of surface photocurrents between two surface states in $\mathrm{Bi}_{2} \mathrm{Te}_{2} \mathrm{Se}$ by 1.5-eV laser pulse. (c) First-principles electronic structure of a seven-formula-units slab of $\mathrm{Bi}_{2} \mathrm{Te}_{2} \mathrm{Se}$ with occupied and unoccupied topological surface state indicated in blue and red arrows (adapted from Ref. [17]).

In the TARPES studies on BTS $[8,13]$ the other unoccupied TSS at around $1.3 \mathrm{eV}$ above the Fermi level was observed. Later, a surface photovoltaic shift of the occupied TSS by about $100 \mathrm{meV}$ was reported, with a long recovery time, exceeding $4 \mu \mathrm{s}$ [14]. Motivated by these studies, here we present a detailed account of the unoccupied electronic structure of BTS including both the lower and the upper TSS with the high spectral resolution using the $1.5-\mathrm{eV}$ photons. We will discuss the ultrafast dynamics of the unoccupied surface resonance and of the upper TSS populated by the resonant optical transitions due to the $1.5-\mathrm{eV}$ laser pulse.

\section{EXPERIMENT}

Single-crystalline samples of BTS were grown by the modified vertical Bridgman method as described elsewhere [25]. The TARPES measurements were conducted using a pulsed laser source equipped with a hemispherical photoelectron analyzer (VG-Scienta R4000) at the Institute for Solid State Physics (ISSP) of the University of Tokyo. The laser pulse (1.48-eV photon energy and 170-fs duration) delivered from a Ti:sapphire laser system operating at $250 \mathrm{kHz}$ repetition (Coherent RegA 9000) was split into two: One pulse is used as a pump, and the other was upconverted into $5.92 \mathrm{eV}$ by using two nonlinear crystals $\beta-\mathrm{BaB}_{2} \mathrm{O}_{4}(\mathrm{BBO})$ and used as a probe. The energy resolution and the Fermi energy position $\left(E_{\mathrm{F}}\right)$ were determined by recording the Fermi cutoff of Au in electrical contact to the sample and the analyzer. The pump-probe delay time was controlled by a delay stage. The delay origin $(t=0)$ and time resolution (380 fs) were determined by recording the pump-probe photoemission signal of HOPG attached next to the sample [26]. Samples were carefully oriented and cleaved in situ at ultrahigh vacuum better than $1 \times 10^{-8} \mathrm{~Pa}$ and afterwards transferred to the main chamber where they were cooled to $7 \mathrm{~K}$ for measurements. Spectral broadening due to the space charge effect was set to $\leqslant 10 \mathrm{meV}$.

\section{RESULTS AND DISCUSSIONS}

Figure 1 presents typical TARPES spectra of BTS along the $\bar{\Gamma} \bar{K}$ and $\bar{\Gamma} \bar{M}$ directions recorded after pumping. Figure 1(a) shows the energy-momentum distribution of the photoemission intensity from both the stationarily occupied states down to $-0.39 \mathrm{eV}$ and the transiently occupied states up to $1.5 \mathrm{eV}$. The high-energy resolution allows to clearly see the sharper features due to the lower and the upper conelike TSSs and the broader features due to the bulk states. Here, the pumpprobe delay time $t$ is set to zero. Let us first focus on the band dispersion along $\bar{\Gamma} \bar{K}$ in the left panel of Fig. 1(a). The lower Dirac point is located at $-0.31 \mathrm{eV}$, while the upper one is at $1.22 \mathrm{eV}$ above the Fermi energy, in accord with the previous studies $[8,16]$. Hereafter, we take the Fermi level to be zero on the energy scale. The lower TSS maintains its linear dispersion above the Fermi level and further above the band gap, remaining distinct from the bulk conduction band (BCB) but getting closer to it at higher energies. The lower $\mathrm{BCB}$ starts at $0.1 \mathrm{eV}$ and disperses parabolically towards higher energies. Furthermore, a projected bulk band gap around the $\bar{\Gamma}$ point is clearly seen between 0.3 and $0.8 \mathrm{eV}$. Interestingly, the upper TSS can be found about $1.5 \mathrm{eV}$ above the lower TSS populated by the laser pulse of $\hbar \omega=1.5 \mathrm{eV}$. This is advantageous for the efficient generation of the spin-polarized photocurrent by the direct photoexcitation of the lower TSS, which is stationarily occupied in an $n$-type topological insulator; see Fig. 1(b). 
(a) $\mathrm{E}-\mathrm{E}_{\mathrm{F}}=0 \mathrm{eV}$
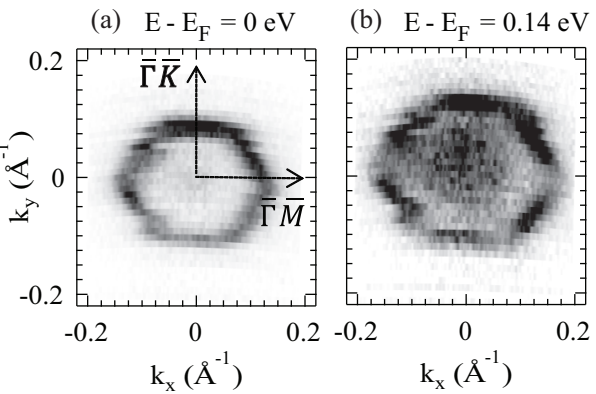

(g) $\mathrm{E}-\mathrm{E}_{\mathrm{F}}=0.26 \mathrm{eV}$

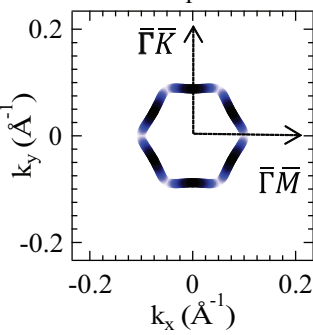

(h) $\mathrm{E}-\mathrm{E}_{\mathrm{F}}=0.4 \mathrm{eV}$

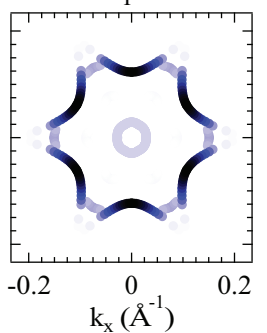

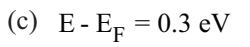

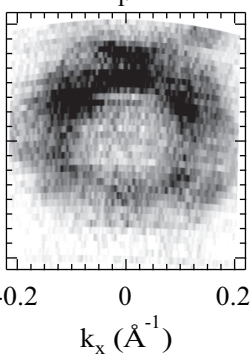

(i) $\mathrm{E}-\mathrm{E}_{\mathrm{F}}=0.56 \mathrm{eV}$

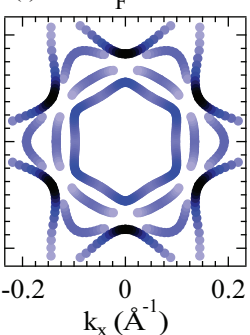

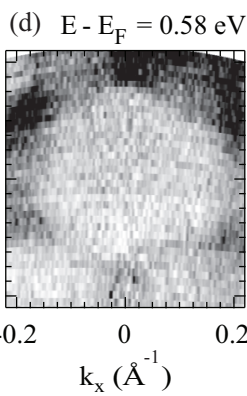
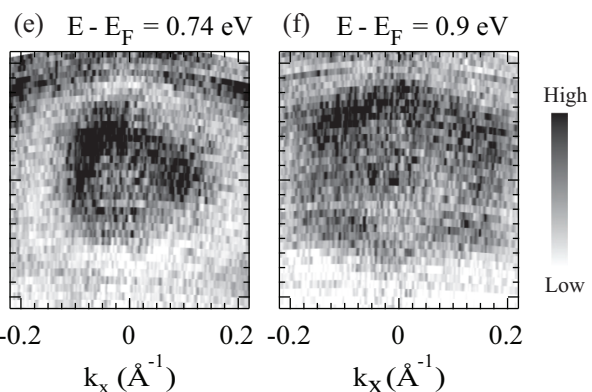

(j) $\mathrm{E}-\mathrm{E}_{\mathrm{F}}=0.84 \mathrm{eV}$

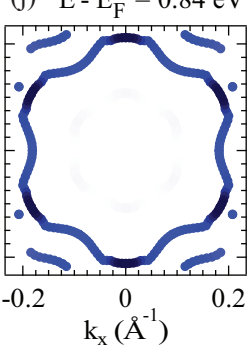

(k) $\mathrm{E}-\mathrm{E}_{\mathrm{F}}=1.0 \mathrm{eV}$

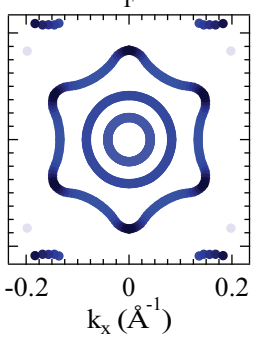

(1) $\mathrm{E}-\mathrm{E}_{\mathrm{F}}=1.16 \mathrm{eV}$

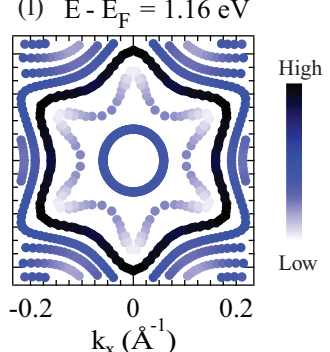

FIG. 2. (a)-(f) Constant energy maps of the angular distribution of photoelectrons. (g)-(l) Spatially resolved Fermi surface. The color scale shows the constant energy cuts of the surface calculated spectral function $N\left(z, k_{\|}\right)$. Energies are referenced to the Fermi level and a $0.26-\mathrm{eV}$ energy shift has been considered between TARPES and calculation.

The right panel of Fig. 1(a) shows the band dispersion along $\bar{\Gamma} \bar{M}$, which has an electronic structure similar to the $\bar{\Gamma} \bar{K}$ direction, only the Dirac point shifts downwards by about $0.04 \mathrm{eV}$ because of the aging effect. It is noteworthy that even though the dispersion of the first TSS is linear between the Dirac point and $E_{F}$, the dispersion starts strongly deviating from linear just above the $E_{F}$, which signifies an outward warped Dirac cone. This confirms our previous result that a strong warping of CECs occurs only along the $\bar{\Gamma} \bar{M}$ direction [16].

In Fig. 1(c) we show for comparison the calculated electronic structure of a seven-formula-units slab of BTS [16,27]. The lower TSS and the conduction bands are well reproduced to within an energy offset of $0.26-0.3 \mathrm{eV}$. In the experiment, the dispersion of the upper TSS is almost perfectly linear, while in the calculation it shows a slight parabolic curvature.

The evolution of the CECs in the unoccupied region is shown in Fig. 2, which compares the measured CECs, Figs. 2(a)-2(f), with the calculated maxima of the momentum distribution of the spatially resolved spectral density $N\left(E, k_{\|}\right)$ at the corresponding energies, Figs. 2(g)-2(1). (The constant energy shift of $0.26 \mathrm{eV}$ of the calculated CECs relative to the measured ones is taken into account.) At the Fermi energy, one can see a hexagonal Fermi contour of the lower TSS, which steadily increases with energy. A small circular contour due to the $\mathrm{BCB}$ emerges at $0.14 \mathrm{eV}$. The outer pattern becomes more warped and broadens at $\sim 0.3 \mathrm{eV}$, while the inner one turns into a hexagon rotated by $90^{\circ}$ relative to the outer pattern. The inner pattern has a contribution from the SRS discovered in our previous work [17]. At $0.74 \mathrm{eV}$, another inner pattern appears due to the bulk band that surrounds the upper TSS, and it changes with energy in a rather irregular way.

The information about the relaxation of the optically excited electrons in BTS can be inferred from the transient ARPES spectra along $\bar{\Gamma} \bar{M}$ for pump-probe delay times up to $24.8 \mathrm{ps}$; see Fig. 3. In the before-pump map $(t=-1.33 \mathrm{ps})$, one sees only the Dirac cone of the lower TSS abruptly cut off at the Fermi level. Upon the arrival of the pump pulse the electrons are excited to the unoccupied states and then gradually relax to the equilibrium state, as seen in the spectra for larger delay times. Note that the BCB splits into two branches with different dispersion and different relaxation behavior, especially for $t<5 \mathrm{ps}$ (in order to better visualize the SRS at $t=0.07 \mathrm{ps,}$ its second derivative image for the selected area is shown in the left panel). This provides further evidence for the existence of the inner surface resonance, which splits off from the top of the lower conduction band (and lies inside the bulk band contour). The surface feature can be clearly discerned by its linear dispersion and the sharp intensity distribution, in contrast to the outer band, whose broader distribution implies its bulk character. This confirms that here we directly observe the SRS (whose intensity distribution overlaps with that of the BCB), and its energy location and dispersion agrees well with our calculated band structure; see Fig. 1(c). The upper TSS has an extremely short recovery time (less than $1 \mathrm{ps}$ ); apparently, owing to the narrow energy band gap, the nonequilibrium electrons rapidly relax to the lower energies. In sharp contrast to the upper TSS, the SRS and the lower TSS exhibit relatively long recovery time. The excited electrons transfer from the higher- to the lower-energy side of the SRS in $\sim 5 \mathrm{ps}$ and then accumulate at the BCB. The electrons at the bottom of $\mathrm{BCB}$ remain there for $\sim 25 \mathrm{ps}$, during which time they relax to constantly populate the lower TSS. The previous study by Neupane et al. [14] claims that the surface photovoltaic (SPV) shift of $100 \mathrm{meV}$ lasts more than $4 \mu \mathrm{s}$. However, it should be noted that the recovery time of SPV is totally different from the recovery time for the transient electrons which is discussed in our case. The surface photovoltage typically occurs in the 


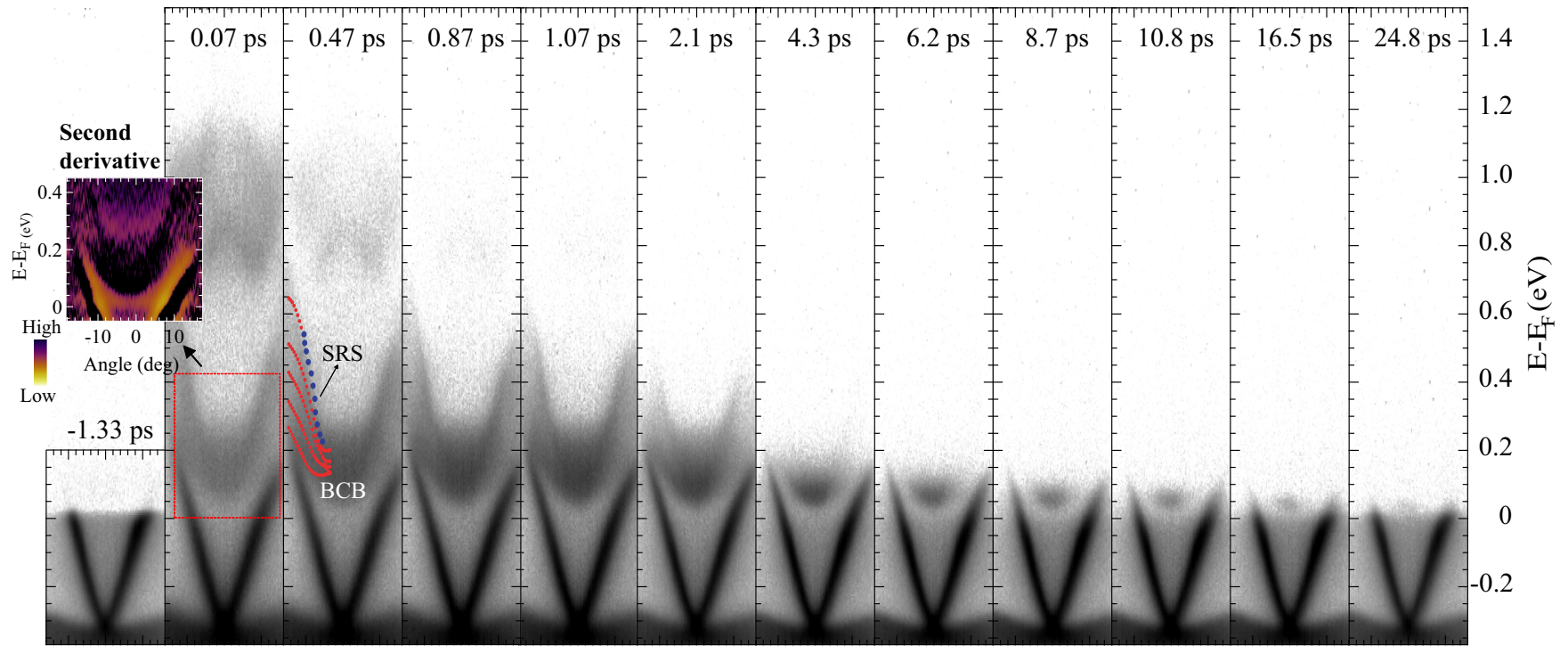

$\begin{array}{llllllllllllllllllllllllllllllllllll}-10 & 0 & 10 & -10 & 0 & 10 & -10 & 0 & 10 & -10 & 0 & 10 & -10 & 0 & 10 & -10 & 0 & 10 & -10 & 0 & 10 & -10 & 0 & 10 & -10 & 0 & 10 & -10 & 0 & 10 & -10 & 0 & 10 & -10 & 0 & 10\end{array}$ Emission angle (deg)

FIG. 3. TARPES spectrum of $\mathrm{Bi}_{2} \mathrm{Te}_{2}$ Se recorded along the $\bar{\Gamma} \bar{M}$ direction with various delay times. The delay time is noted on the spectra. Second derivative of the selected area in $t=0.07 \mathrm{ps}$ is shown in the left panel for better visualizing of the SRS.

highly bulk-insulating samples, where the band bending near the surface is flattened by the creation of electron-hole pairs, which leads to the shift of the TSS caused by pumping. At the same time, no significant SPV is observed in the present work, which might be due to a less insulating bulk than in the experiment of Ref. [14].

To see more clearly the energy-dependent carrier dynamics in the surface and in the bulk bands, let us follow how
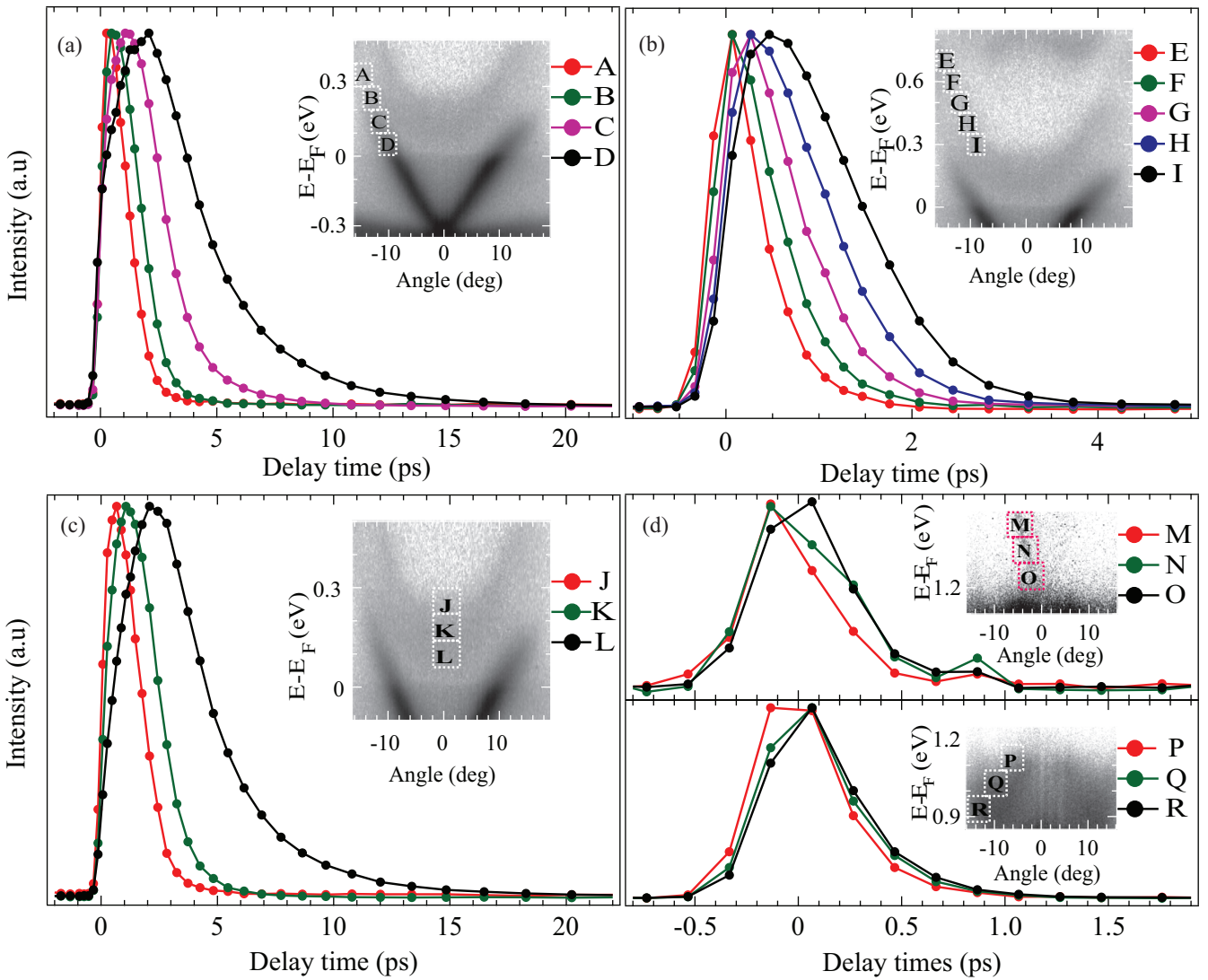

FIG. 4. Ultrafast time evolution of the first and second topological surface state as well as the bulk conduction band population in corresponding frames set (see inset). 
the intensity profiles vary with delay time in the energymomentum frames A-R; see Figs. 4(a)-4(d). The intensity is normalized to the peak amplitude of each frame. It turns out that the profile of the decay curve depends on the frame, while there is a general trend that the depletion rate decreases with decreasing energy (i.e., with approaching the Fermi level). Let us first compare the regions A-D with the regions E-I, see Figs. 4(a) and 4(b), which will help us understand the interplay between TSS and SRS. The intraband scattering plays the main role in the relaxation of these states, so the SRS recovers faster (in about $5 \mathrm{ps}$ ) than the lower TSS (in more than $15 \mathrm{ps}$ ). Next we consider the electron dynamics in the BCB, see Fig. 4(c), where the recovery time is longer than in SRS, and the nonequilibrium occupation persists for more than $15 \mathrm{ps}$. Similar to the $p$-type $\mathrm{Bi}_{2} \mathrm{Se}_{3}$ [7], the decay rates of the TSS and BCB are obviously different, which means that there is an additional channel through which the surface state is filled. Because further decay is hampered by the presence of the band gap, the electrons pile up at the bottom of the conduction band, and after 10 ps only the bottom of the BCB remains populated above the Fermi level. Thus, a metastable population is formed at the bottom of the $\mathrm{BCB}$ with a long relaxation time comparable to the TSS (more than $15 \mathrm{ps}$ ); see frames D and L in Figs. 4(a) and 4(c), respectively. The slow decay of the metastable population of the BCB suggests that at larger times the lower-lying TSS is being constantly filled from this source. Finally, let us consider the dynamics of the upper TSS and the bulk band at this energy; see Fig. 4(d). Note that, different from other energy states, the transient intensity profiles of these states are almost symmetric with respect to $t=0$ because of the negligible influx of electrons into these states from higher energies. The photoexcited electrons in these states are very short-lived, and they rapidly relax to the lower TSS or transfer into the more stable lower bulk states. As a result, a very short recovery time of around $1 \mathrm{ps}$ is observed for the upper TSS.

\section{CONCLUSION}

To summarize, we have presented a detailed time- and angle-resolved photoemission study of the unoccupied band structure and ultrafast nonequilibrium dynamics of $\mathrm{Bi}_{2} \mathrm{Te}_{2} \mathrm{Se}$, including both the lower and the upper topological surface states. We have directly observed the unoccupied surface resonance state that overlaps with the bulk conduction band. Most important, we demonstrated the feasibility of the direct optical transitions between the two TSSs: we have efficiently populated the upper TSS with the $1.5-\mathrm{eV}$ radiation of the Ti:sapphire laser source. Furthermore, we observed the metastable population at the $\mathrm{BCB}$ edge that lasted longer than $15 \mathrm{ps}$ and established that the metastable electrons in this band decay to continuously fill the TSS. The present findings may open more perspectives for designing the topologicalinsulator-based photodetectors and optical devices.

\section{ACKNOWLEDGMENTS}

The TARPES measurements were jointly carried out by the Laser and Synchrotron Research Center of the Institute for Solid State Physics of at the University of Tokyo. This work was financially supported by KAKENHI (Grants No. 17H06138 and No. 26800165) and the bilateral collaboration program between RFBR (Russia; Grant No. 15-52-50017) and JSPS (Japan). This work also was supported by the Spanish Ministry of Economy and Competitiveness MINECO (Project No. FIS2016-76617-P). O.E.T. and K.A.K. were supported by the Russian Science Foundation (Project No. 17-12-01047).
[1] L. Fu, C. L. Kane, and E. J. Mele, Phys. Rev. Lett. 98, 106803 (2007).

[2] D. Hsieh, Y. Xia, D. Qian, L. Wray, J. H. Dil, F. Meier, J. Osterwalder, L. Patthey, J. G. Checkelsky, N. P. Ong, A. V. Fedorov, H. Lin, A. Bansil, D. Grauer, Y. S. Hor, R. J. Cava, and M. Z. Hasan, Nature 460, 1101 (2009).

[3] P. Roushan, J. Seo, C. V. Parker, Y. S. Hor, D. Hsieh, D. Qian, A. Richardella, M. Z. Hasan, R. J. Cava, and A. Yazdani, Nature 460, 1106 (2009).

[4] H. Beidenkopf, P. Roushan, J. Seo, L. Gorman, I. Drozdov, Y. S. Hor, R. J. Cava, and A. Yazdani, Nat. Phys. 7, 939 (2011).

[5] M. Z. Hasan and J. E. Moore, Annu. Rev. Condens. Matter Phys. 2, 55 (2011).

[6] T. Okuda and A. Kimura, J. Phys. Soc. Jpn. 82, 021002 (2013).

[7] J. A. Sobota, S. Yang, J. G. Analytis, Y. L. Chen, I. R. Fisher, P. S. Kirchmann, and Z. X. Shen, Phys. Rev. Lett. 108, 117403 (2012).

[8] D. Niesner, Th. Fauster, S. V. Eremeev, T. V. Menshchikova, Y. M. Koroteev, A. P. Protogenov, E. V. Chulkov, O. E. Tereshchenko, K. A. Kokh, O. Alekperov, A. Nadjafov, and N. Mamedov, Phys. Rev. B 86, 205403 (2012).
[9] Y. H. Wang, D. Hsieh, E. J. Sie, H. Steinberg, D. R. Gardner, Y. S. Lee, P. Jarillo-Herrero, and N. Gedik, Phys. Rev. Lett. 109 127401 (2012).

[10] M. Hajlaoui, E. Papalazarou, J. Mauchain, G. Lantz, N. Moisan, D. Boschetto, Z. Jiang, I. Miotkowski, Y. P. Chen, A. TalebIbrahimi, L. Perfetti, and M. Marsi. Nano Lett. 12, 3532 (2012).

[11] J. A. Sobota, S. L. Yang, A. F. Kemper, J. J. Lee, F. T. Schmitt, W. Li, R. G. Moore, J. G. Analytis, I. R. Fisher, P. S. Kirchmann, T. P. Devereaux, and Z. X. Shen, Phys. Rev. Lett. 111, 136802 (2013).

[12] J. A. Sobota, S. L. Yang, D. Leuenberger, A. F. Kemper, J. G. Analytis, I. R. Fisher, P. S. Kirchmann, T. P. Devereaux, and Z. X. Shen, J. Electron Spectrosc. Relat. Phenom. 195, 249 (2014).

[13] D. Niesner, S. Otto, Th. Fauster, E. V. Chulkov, S. V. Eremeev, O. E. Tereshchenko, and K. A. Kokh, J. Electron Spectrosc. Relat. Phenom. 195, 258 (2014).

[14] M. Neupane, S. Y. Xu, Y. Ishida, S. Jia, B. M. Fregoso, C. Liu, I. Belopolski, G. Bian, N. Alidoust, T. Durakiewicz, V. Galitski, S. Shin, R. J. Cava, and M. Z. Hasan, Phys. Rev. Lett. 115, 116801 (2015). 
[15] S.Y. Zhu, Y. Ishida, K. Kuroda, K. Sumida, M. Ye, J. J. Wang, H. Pan, M. Taniguchi, S. Qiao, S. Shin, and A. Kimura, Sci. Rep. 5, 13213 (2015).

[16] D. Niesner, S. Otto, V. Hermann, Th. Fauster, T. V. Menshchikova, S. V. Eremeev, Z. S. Aliev, I. R. Amiraslanov, M. B. Babanly, P. M. Echenique, and E. V. Chulkov, Phys. Rev. B 89, 081404(R) (2014).

[17] M. Nurmamat, E. E. Krasovskii, K. Kuroda, M. Ye, K. Miyamoto, M. Nakatake, T. Okuda, H. Namatame, M. Taniguchi, E. V. Chulkov, K. A. Kokh, O. E. Tereshchenko, and A. Kimura, Phys. Rev. B 88, 081301(R) (2013).

[18] C. Cacho, A. Crepaldi, M. Battiato, J. Braun, F. Cilento, M. Zacchigna, M. C. Richter, O. Heckmann, E. Springate, Y. Liu, S. S. Dhesi, H. Berger, Ph. Bugnon, K. Held, M. Grioni, H. Ebert, K. Hricovini, J. Minár, and F. Parmigiani, Phys. Rev. Lett. 114, 097401 (2015).

[19] C. Jozwiak, J. A. Sobota, K. Gotlieb, A. F. Kemper, C. R. Rotundu, R. J. Birgeneau, Z. Hussain, D. H. Lee, Z. X. Shen, and A. Lanzara, Nat. Commun. 7, 13143 (2016).
[20] J. W. Mclver, D. Hsieh, H. Steinberg, P. Jarillo-Herrero, and N. Gedik, Nat. Nanotechnol. 7, 96 (2012).

[21] J. Xiong, A. C. Petersen, D. X. Qu, Y. S. Hor, R. J. Cava, and N. P. Ong, Physica E 44, 917 (2012).

[22] J. G. Checkelsky, Y. S. Hor, M. H. Liu, D. X. Qu, R. J. Cava, and N. P. Ong, Phys. Rev. Lett. 103, 246601 (2009).

[23] J. G. Analytis, J. H. Chu, Y. L. Chen, F. Corredor, R. D. McDonald, Z. X. Shen, and I. R. Fisher, Phys. Rev. B 81, 205407 (2010).

[24] G. Wang, X. G. Zhu, Y. Y. Sun, Y. Y. Li, T. Zhang, J. Wen, X. Chen, K. He, L. L. Wang, X. C. Ma, J. F. Jia, S. B. Zhang, and Q. K. Xue, Adv. Mater. 23, 2929 (2011).

[25] K. A. Kokh, B. G. Nenashev, A. E. Kokh, and G. Y. Shvedenkov, J. Cryst. Growth 275, e2129 (2005).

[26] Y. Ishida, T. Togashi, K. Yamamoto, M. Tanaka, T. Kiss, T. Otsu, Y. Kobayashi, and S. Shin, Rev. Sci. Instrum. 85, 123904 (2014).

[27] Self-consistent calculations within the local density approximation were performed with the full-potential augmented plane wave method described in E. E. Krasovskii, F. Starrost, and W. Schattke, Phys. Rev. B 59, 10504 (1999). 